\title{
Emulsion stability and antimicrobial activity of Ionic liquid-based formulation.
}

\author{
Mansoor U1 Hassan Shah ${ }^{1,2}$, Muhammad Moniruzzaman ${ }^{1,2,}$, Mahabubur Rahman Talukder ${ }^{3}$, and Suzana Yusup ${ }^{1}$ \\ ${ }^{1}$ Department of Chemical Engineering, Universiti Teknologi PETRONAS, 32610, Bandar Seri Iskandar, Perak, Malaysia \\ ${ }^{2}$ Centre of Research in Ionic Liquids (CORIL), Universiti Teknologi PETRONAS, 32610, Bandar Seri Iskandar, Perak, Malaysia \\ ${ }^{3}$ Institute of Chemical and Engineering Sciences (ICES), Agency for Science, Technology and Research, 627833, 1 Pesek Road, Jurong \\ Island, Singapore.
}

\begin{abstract}
Chemical dispersants is one of the globally accepted remediation technique used for marine oil spill. However, the toxicity related with these dispersants confined its application in marine environment. Therefore, to overcome this problem, the employment of environmentally benign dispersants is one of the effective conceivable approach. In this study, the formulation comprised of choline based ionic liquid, choline laurate ([Cho][Lau]) and a biosurfactant, lactonic sophorolipids (LS) were used as a crude oil emulsifier. The toxicity of the newly developed formulation was evaluated to confirm their safe employment in sea water. The developed formulation worked effectively as a crude oil emulsifier and formed a stable crude oil emulsion. The toxicity study against Gram-positive and -negative bacteria depicts the "practically harmless" nature of the developed formulation. Thus, the results presented in this study showed that the new formulation can potentially replace the conventional dispersant used for marine oil spill remediation.
\end{abstract}

Key words: Ionic liquids; biosurfactant; oil spill; formulation; toxicity.

\section{Introduction}

The marine eco system serves as a natural sink for the accidental or deliberate release of petroleum hydrocarbons due to several reasons including; drilling, storing, tanker accidents, and waste management [1]. The Deepwater horizon, one of the major oil spill had transferred huge amount of crude oil ( $>$ two hundred million gallons ) to the Mexico Gulf and have harmful impact on the coastal and marine eco system [2]. To reduce the consequences of such disastrous oil spill, several response techniques were used and among all these techniques, chemical dispersants was found to be one of the most appropriate method that minimizes the damages caused by the oil spills [3].

The use of chemical dispersants for the marine oil spill remediation is comprehensively reported globally however, the toxicity issues related with the most wellknown available commercial dispersants, restricted many governmental organizations to use it in marine environment [4]. For instance, Corexit was the most frequently employed major oil spill dispersant however, the prominent component of Corexit i.e., dioctyl sodium sulfosuccinate (DOSS) and the solvent used in its formulation was found to be non-biodegradable and toxic $[4,5]$. Therefore, the growing environmental concerns and strict rules made by concern authorities motivated the researchers and scientists to formulate alternative chemical dispersants that are nontoxic and environmentally benign $[6,7]$.

Biosurfactants (BS) emerges as a promising candidate for environmental remediation due to its high biodegradability and low toxicity [8]. However, the tediousness in large scale production of BS is one of the considerable limitation to use BS effectively for environmental applications [9, 10]. Hence, BS are normally combined with inexpensive synthetic surfactants. Even though the merging of synthetic surfactants with BS is useful in terms of cost and surface properties but their existence result in formulations with high toxicity. Hence, a nontoxic amphiphilic component is required that can merge with BS to treat marine oil spills by a greener approach.

Recently, ionic liquids (ILs) have garnered high consideration due to their favorable technological and environmental advantages of low toxicity, low volatility, low flammability and high surface activity [11, 12]. Moreover, the self-assembling behavior of surface active ILs have provided the opportunity to utilize these amphiphiles effectively for chemical and environmental applications [13]. In recent times, choline based ILs have gained high attention due to their less toxic and high

* Corresponding author: m.moniruzzaman@utp.edu.my 
biodegradable nature [13-15]. Therefore, these environmentally benign surfactants offer a new gate way to be used for environmental remediations $[14,16]$. In our recent study [17], we have investigated the emulsifying property of the newly developed formulation composed of choline laurate, [Cho][Lau] and lactonic sophorolipids, (LS) and found that the formulation effectively emulsify the light Tapis crude oil. These observations motivated us to further evaluate the ability of this new formulation to emulsify the medium Arab crude oil. It would be interesting to see how this formulation emulsify a range of crude oils (light and medium crude oil). Moreover, to explore their safe employment in sea, their toxicity by using different microorganisms were also evaluated.

\section{Materials and Methods}

\subsection{Materials}

In the current research study, the sophorolipids employed were in lactonic forms. The detailed procedure regarding the production of sophorolipids and its subsequent purification to obtain the pure lactonic form $(>99 \%)$ were mentioned in our previous studies [7, 17]. Similarly the choline based ILs was also synthesized in our laboratory by a method explained in our previous study [13].

\subsection{Emulsion preparation}

Synthetic seawater (using Instant Ocean sea salt) and Arab crude oil emulsions were prepared by adding $1 \mathrm{~mL}$ Arab crude oil to $10 \mathrm{~mL}$ synthetic sea water. A dispersant $(1 \mathrm{~mL})$ was added on the surface of crude oil, and then a vortex mixer (IKA Vortex Genius 3) at $3000 \mathrm{rpm}$ were used to shake the sample for 5 minutes. The vials were then observed visually to investigate the stability of the formed emulsion by taking images over a period of time.

\subsection{Microorganisms}

The toxicity of the newly developed formulations were evaluated against different bacteria. The tested bacteria were Gram-positive and Gram negative (Staphylococcus aureus S 1426 and Listeria monocytogenes L 49, Escherichia coli E 149 and Aeromonas hydrophila A 97) respectively, provided by Institute of Medical Research, Kuala Lumpur, Malaysia.

\subsection{Antimicrobial activity assessment}

The antimicrobial activity of the binary mixtures of LS and ILs were evaluated by employing the standard broth tube dilution approach [18]. By using 96-well plates, twofold serial dilution were carried out for pure components as well their mixtures. Mueller-Hinton broth were used for bacterial growth by injecting the bacterial colonies from agar plates to the medium (Mueller-Hinton). The optimum growth of bacteria was then confirmed by measuring the optical density after $24 \mathrm{~h}$ of incubation. Once the optimum growth of bacteria was achieved, 100 $\mu \mathrm{L}$ of Mueller-Hinton broth were transferred to row second (2) to eight (8) of the 96-well plates. Afterwards, $100 \mu \mathrm{L}$ of tested sample were transferred to the first and second rows respectively. The two-fold dilution was performed from the second to seventh row. The eighth row (last) were used as a control where only pure media were added. Finally, the bacterial suspension solution $(100 \mu \mathrm{L})$ were added to each row of the 96 -well plates. The plates were then placed for $24 \mathrm{~h}$ in the incubator at 37 ${ }^{\circ} \mathrm{C}$. The absorbance at $620 \mathrm{~nm}$ were recorded by employing the micro-plate reader and the respective $\mathrm{EC}_{50}$ values were determined by a dose response curve.

\section{Results and discussion}

\subsection{Assessment of crude oil emulsion}

To investigate the application of the developed formulation as crude oil emulsifier, the crude oil emulsions were made at 1:10 (v/v) of oil-to-seawater. For all experiments the dispersant-to-oil ratio (DOR) was kept at $1: 1(\mathrm{v} / \mathrm{v})$. The emulsion results of the individual surfactant as well of the binary mixture containing 40:60 $(\mathrm{w} / \mathrm{w})$ ratio of [Cho][Lau] and LS are presented in Fig. 1. In our previous study [17] we already found that the micelles formed at the above stated ratio are more thermodynamically stable and had the capability to reduce the interfacial tension to a lower value. Therefore, the same ratio is selected in the present study to investigate the emulsion stability formed by using a medium Arab crude oil. The physicochemical properties of the Arab crude oil are tabulated in Table 1.

Table 1. The physicochemical properties of Arab crude oil.

\begin{tabular}{|c|c|c|}
\hline Properties & Unit & Values \\
\hline API Gravity & API & 27.70 \\
\hline Density $\left(20^{\circ} \mathrm{C}\right)$ & $\mathrm{Kg} / \mathrm{L}$ & 0.902 \\
\hline Viscosity $\left(25^{\circ} \mathrm{C}\right)$ & $\mathrm{cSt}$ & 53.36 \\
\hline HPLC - Saturates & $\mathrm{Wt} \%$ & 3.92 \\
\hline HPLC - Aromatics (Total) & $\mathrm{Wt} \%$ & 70.15 \\
\hline
\end{tabular}

For the analysis of emulsion stability, three cases of crude oil emulsions were studied. The first two cases illustrated the single component emulsions (Fig. 1A, 1B) whereas the third case represented the emulsion formed by 40:60 (w/w) ratio of [Cho][Lau] and LS (Fig. 1C). In the first two cases (at $\mathrm{t}=0$ minutes), homogeneous emulsions were produced after the mixing at $3000 \mathrm{rpm}$. However, after 30 minutes of mixing, in both cases the dispersed crude oil rises to the top surface of sea water, depicting the unstable nature of the emulsion. However, the emulsion produced by the individual LS (Fig. 1B) 
exhibited high stability for a longer span of time than individual [Cho][Lau]. On the other side, the emulsion formed by the developed formulation (40:60 ratio of [Cho][Lau] and LS and) (Fig. 1C) was almost similar at $t$ $=0$ minutes and $t=30$ minutes, indicating more stable emulsion formation as compared to the first two cases. Moreover, for the third case, the developed formulation was able to produce emulsion which was stable when observed even after $18 \mathrm{hr}$ of mixing time, illustrating a very low degree of coalescence and creaming (Fig. 1C). Hence, all these results showed that the developed formulation have the capability to form a more stable emulsion of Arab crude oil in synthetic sea water.

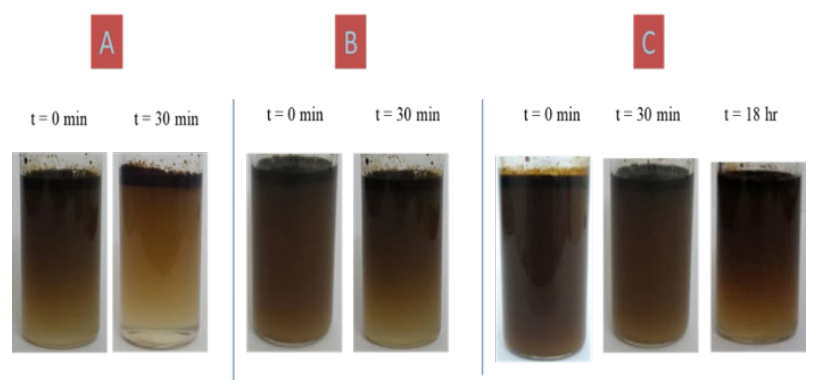

Fig. 1. Emulsion formed between Arab crude oil and sea water: (a) $100 \%$ [Cho][Lau], (b) $100 \%$ LS, and (c) $40 \%$ [Cho][Lau] + $60 \%$ LS.

\subsection{Antimicrobial activity of the developed formulation}

The antimicrobial activity of the [Cho][Lau] and LS mixed surfactant systems were evaluated against four microbial isolates as mentioned in section 2.3. Fig. 2 (a) showed the sub culturing of bacteria on plates containing Luria bertani Aga, whereas Fig. 2 (b) shows the optimum growth of respective bacteria in Mueller-Hinton broth after $24 \mathrm{~h}$ incubation.
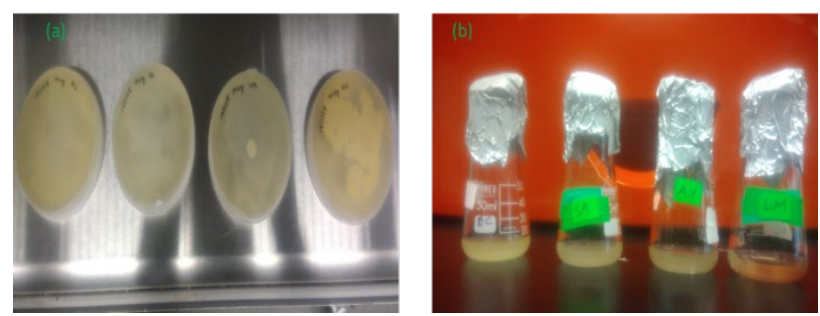

Fig. 2. Bacteria (a) sub-culturing and (b) optimum growth in Mueller-Hinton broth after $24 \mathrm{~h}$ incubation.

The toxicity of binary mixtures containing different mole fraction of LS " $\alpha$ " against four different types of microorganisms were evaluated to ensure its safe nature for marine microorganisms. Fig. 3 showed the $\mathrm{EC}_{50}$ values of the binary mixtures composed of LS and [Cho][Lau] and the obtained $\mathrm{EC}_{50}$ values were then compared with the toxicity scale developed by Passino and Smith [19] as shown in Table 2.

Table 2. Toxicity ranking established by Passino \& Smith [20].

\begin{tabular}{|c|c|}
\hline Relative toxicity & $\mathrm{EC}_{50}\left(\mathrm{mg} \mathrm{L}^{-1}\right)$ \\
\hline Super Toxic & $0.01-0.1$ \\
\hline Highly Toxic & $0.1-1$ \\
\hline Moderately Toxic & $1-10$ \\
\hline Slightly Toxic & $10-100$ \\
\hline Practically harmless & $100-1000$ \\
\hline Harmless & $>1000$ \\
\hline
\end{tabular}

The results presented in Fig. 3 illustrated that the toxicity profiles of all the combination occur in the range of "practically harmless" (Table. 2). As it is reported that the toxicity of the mixture depended on the mixture dose ratio and the existence of an IL having complex anion may affected the toxicity of the system. However, the relation between ILs hydrophobicity and the noted toxic effect is based on unclear interactions with membrane proteins and lipid membranes [21].

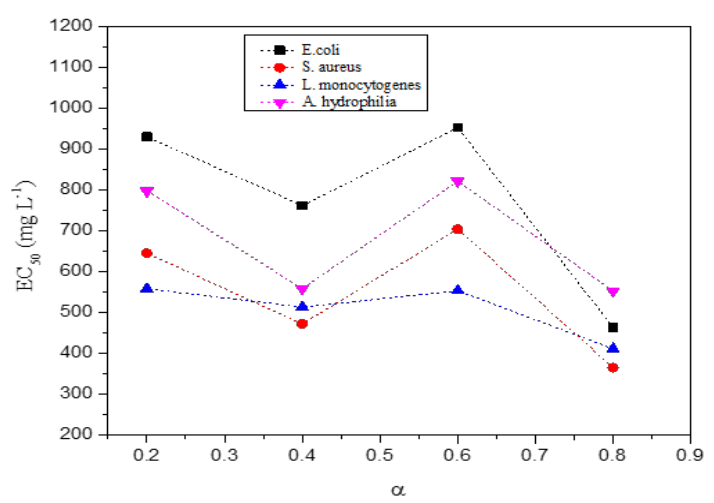

Fig. 3. $E_{50}$ values of binary surfactant systems of LS and [Cho][Lau] against four different types of bacteria.

It is well established that the less or more stable micelles formation may affect the bioavailability of the mixture which in turn effect their biological activity [21]. Further, it is noteworthy that at $\alpha=0.6$ (Fig. 3), the toxicity of the binary mixtures was significantly reduced. Therefore, at this ratio $(\alpha=0.6)$ both the surface-active components of the developed formulations were highly taking part in the micelles formation and its toxicity reduced by the robust interaction between them. Similar results were reported before, where the stable micelle formation reduced the toxicity of the mixture [13]. Thus, the results achieved in the current research illustrated that all the formulations can be safe for marine oil spill as well for other biological applications.

\section{Conclusions}

In the current research study, the emulsification activity and toxicity study of the developed ILs based formulations were carried out. The developed formulations were found to be an effective emulsifier against Arab light oil by forming a stable emulsion. The Antimicrobial activity analysis showed that all the 
developed formulations occurred in the rage of "practically harmless", depicting their non-toxic nature for oil spill remediation.

\section{Acknowledgments}

The present work was supported by the Fundamental Research Grant Scheme (FRGS 015MA0-091) of the Ministry of Higher Education, Malaysia.

\section{References}

1. Z.H. Asadov, A.H. Tantawy, I.A. Zarbaliyeva, R.A. Rahimov, Synthesis of new surface-active ammonium-type complexes based on palmitic acid for removing thin petroleum films from water surface, Egypt. J. Pet., 22 (2013).

2. R. M. Atlas and T. C. Hazen, "Oil Biodegradation and Bioremediation: A Tale of the Two Worst Spills in US History," Environ Sci Technol, vol. 45 (2011).

3. H. Chapman, K. Purnell, R.J. Law, M.F. Kirby, The use of chemical dispersants to combat oil spills at sea: A review of practice and research needs in Europe, Mar. Pollut. Bull., 54 (2007).

4. R. Almeda, C. Hyatt, E.J. Buskey, Toxicity of dispersant Corexit 9500A and crude oil to marine microzooplankton, Ecotoxicol. Environ. Saf. 106 (2014).

5. E. Nyankson, M.J. DeCuir, R.B. Gupta, Soybean lecithin as a dispersant for crude oil spills, ACS Sustain. Chem. Eng., 3 (2015).

6. R. Maier, G. Soberon-Chavez, Pseudomonas aeruginosa rhamnolipids: biosynthesis and potential applications, Appl. Microbiol. Biotechnol. 54 (2000).

7. M.U.H. Shah, M. Sivapragasam, M. Moniruzzaman, M.M.R. Talukder, S.B. Yusup, M. Goto, Production of sophorolipids by Starmerella bombicola yeast using new hydrophobic substrates, Biochem. Eng. J. 127 (2017).

8. I.N. Van Bogaert, K. Saerens, C. De Muynck, D. Develter, W. Soetaert, E.J. Vandamme, Microbial production and application of sophorolipids, Appl. Microbiol. Biotechnol. 76 (2007).

9. S.A. Onaizi, M. Nasser, F.A. Twaiq, Micellization and interfacial behavior of a synthetic surfactant-biosurfactant mixture, COLLOID SURFACE A, 415 (2012).

10. Z. Najmi, G. Ebrahimipour, A. Franzetti, I. Banat, In situ downstream strategies for costeffective biosurfactant recovery, BIOTECHNOL APPL BIOC 65 (2018).

11. A.M. Atta, H.A. Al-Lohedan, M.M. Abdullah, S.M. ElSaeed, Application of new amphiphilic ionic liquid based on ethoxylated octadecylammonium tosylate as demulsifier and petroleum crude oil spill dispersant, J IND ENG CHEM, 33 (2016).

12. M.U.H. Shah, M. Sivapragasam, M. Moniruzzaman, M.M.R. Talukder, S.B. Yusup, Dispersion of crude oil by choline based ionic liquids, Materials Today: Proceedings, 5 (2018).

13. M.U.H. Shah, M. Sivapragasam, M. Moniruzzaman, M.M.R. Talukder, S.B. Yusup, M. Goto, Aggregation behavior and antimicrobial activity of a micellar system of binary ionic liquids, J. Mol. Liq, 266 (2018).

14. B.L. Gadilohar, G.S. Shankarling, Choline based ionic liquids and their applications in organic transformation, J. Mol. Liq, 227 (2017).

15. M.U.H. Shah, M. Moniruzzaman, M.M.R. Talukder, S.B. Yusup, Antimicrobial Activity and Acute Toxicity of Choline Based Ionic Liquid Mixtures, Materials Today: Proceedings, 19 (2019).

16. J. Aburto, D.M. Márquez, J.C. Navarro, R. Martínez-Palou, Amphiphilic Choline Carboxylates as Demulsifiers of Water-in-Crude Oil Emulsions, TENSIDE SURFACT DET, 51 (2014).

17. M.U.H. Shah, M. Moniruzzaman, M. Sivapragasam, M.M.R. Talukder, S.B. Yusup, M. Goto, A binary mixture of a biosurfactant and an ionic liquid surfactant as a green dispersant for oil spill remediation, J. Mol. Liq. 280 (2019).

18. A. Foulet, O.B. Ghanem, M. El-Harbawi, J.-M. Lévêque, M.A. Mutalib, C.-Y. Yin, Understanding the physical properties, toxicities and anti-microbial activities of choline-amino acid-based salts: Low-toxic variants of ionic liquids, J. Mol. Liq. 221 (2016)

19. D.R.M. Passino, S.B. Smith, Quantitative structure-activity relationships (QSAR) and toxicity data in hazard assessment, QSAR in Environmental Toxicology-II, Springer (1987).

20. D.R. Passino, S.B. Smith, Acute bioassays and hazard evaluation of representative contaminants detected in Great Lakes fish, ENVIRON TOXICOL CHEM, 6 (1987)

21. S. Steudte, P. Stepnowski, C.-W. Cho, J. Thöming, S. Stolte, (Eco) toxicity of fluoroorganic and cyano-based ionic liquid anions, CHEM COMMUN, 48 (2012). 